\title{
LCLS BEAM DYNAMICS STUDIES WITH THE 3-D PARALLEL IMPACT-T CODE*
}

\author{
Y. Ding ${ }^{\dagger}$, Z. Huang and C. Limborg-Deprey, SLAC, Menlo Park, CA 94025, USA \\ J. Qiang, LBNL, Berkeley, CA 94720, USA
}

\section{Abstract}

We present the beam dynamics studies with the 3-D Parallel Impact-T code on the Linac Coherent Light Source (LCLS) injector system. The physics model and recent additions of the Impact-T code are reviewed, and the space charge simulation studies are described. Simulation results show that for the normal design parameters of the LCLS with a smooth laser profile, the space charge effects above $135 \mathrm{MeV}$ are negligible. However, the electron bunch can form a very-high current spike after the bunch compressor. In this case space charge still play an important role in affecting the beam quality in both longitudinal and transverse directions.

\section{INTRODUCTION}

The Linac Coherent Light Source (LCLS) injector beamline is cuurrently under commissioning at SLAC [1]. It consists of a BNL/SLAC/UCLA 1.6 cell S-band gun followed by two 3-meter long SLAC accelerator sections (linac-0). The electron beam is accelerated to $135 \mathrm{MeV}$ after the injector, then it is injected into the main linac axis via a 35 degree double bend achromatic (DL1). A detailed description of the major injector components can be found in [2]. The electron beam is accelerated to $250 \mathrm{MeV}$ with 3 accelerator sections (linac-1) and an X-band linearizing section. It is then compressed longitudinally in the first bunch compressor (BC1) to an rms length of $190 \mu \mathrm{m}$ based on the standard design parameters with $1 \mathrm{nC}$ total bunch charge.

Space charge is the most important beam dynamics issue in the injector beamline. Impact-T, a 3D beam dynamics code which has a parallel implementation, makes it very useful and fast for space charge studies [3]. A benchmark study between Impact-T and PARMELA codes using an $\mathrm{S}$ band photoinjector at SLAC shows good agreement in the simulation results [4]. In this paper, we use an updated version of Impact-T code to study the space charge effects at relatively higher electron energies up to $250 \mathrm{MeV}$, where the bunch undergoes magnetic compression. We give numerical examples where the space charge effects are still prominent after the bunch compression.

\footnotetext{
* This work was supported by Department of Energy Contracts No. DE-AC02-76SF00515.

† Email: ding@slac.stanford.edu.
}

05 Beam Dynamics and Electromagnetic Fields

\section{IMPACT-T CODE}

The Impact-T code is a three-dimensional parallel particle tracking code to study beam dynamics in photoinjector or linac. It uses a particle-in-cell method to include the space-charge forces of the beam self-consistently [3]. Here, the space-charge forces are included under a quasi-static approximation, that is, the electro-static Poisson equation is solved in the moving beam frame. The electrostatic fields are transformed back to the laboratory frame to obtain both the electric and magnetic fields. The effects of large energy spread can be included by using a multiple slice so that in each slice the relative energy spread is small and the Lorentz transform is valid. Besides space-charge effects, short-range longitudinal and transverse wakefields from accelerating structure are also included in the Impact-T code by the convolution of wake functions and particle distribution. The wake functions of SLAC traveling wave structure follows the approximations by Bane [5]. For the backward traveling wave structure, it uses the approximations given by Craievich et al.[6]. A wake function can also be read in as a discrete data and linearly interpolated to individual macroparticles during the simulation. The effects of coherent synchrotron radiation (CSR) are included through the convolution of a longitudinal CSR wake function and particle distribution. The CSR wake function used here follows the approximations used by Borland [8] and Stupakov et al. [9] from Saldin et al.'s one-dimensional (1D) model [7]. The effects of transient radiation entering and exiting from the bending magnet are also included.

The Impact-T code has a comprehensive set of beamline elements, and furthermore allows arbitrary overlap of their fields, which gives the Impact- $T$ a capability to model both the standing wave structure and traveling wave structure. For an rf cavity with azmuthal symmetry, the electromagnetic fields are obtained from the on-axis electric field profile up to 3 rd order approximation. Here, the on-axis electric field is approximated by a number of Fourier series expansion coefficients. The electromagnetic fields can also be read in as a two-dimensional discrete data or fully threedimensional data during the simulation. The fields in traveling wave structure are modeled using a superposition of of two standing wave fields [10]. The external magnetic focusing elements include solenoid, dipole, quadrupole, sextupole, octupole and decapole. The detailed field profile on axis in a solenoid is used to get the off-axis field up to 3rd order approximation. These fields can also be directly read in from a discrete two-dimensional data file during simulation. The fields inside the bending magnet are divided 
into a constant field region and two fringe field regions at the entrance and the exit. The fringe field on the mid-plane is approximated by an Enge function. The magnetic fields inside a quadrupole magnet are treated as a constant gradient field or including some fringe fields on both ends. The fringe field is also approximated by an two coefficient Enge function. More detailed description of the Impact-T code can be found in Ref. [11].

An additional longitudinal space charge exists for a converging or diverging beam that do not depend on $\gamma$ [14]. Such a force is typically negligible unless the beam sizes change drastically in bunch compressors. At present, this longitudinal force is not modeled in Impact-T. Therefore, we use only the 1D CSR model inside the bunch compressor for consistency.

\section{SIMULATION STUDIES WITH NOMINAL DESIGN PARAMETERS}

Here we describe the LCLS injector beam dynamics studies with Impact-T. Following the nominal design parameters, we use a 10-ps flattop laser profile and a uniform transverse distribution with $1.2 \mathrm{~mm}$ radius to generate $1 \mathrm{nC}$ bunch charge. The peak accelerating gradient of the rf gun is set at $120 \mathrm{MV} / \mathrm{m}$, and the laser launching phase relative to the crest of the rf field is about 32 degree in S-band. The beam energy after the gun is about $6 \mathrm{MeV}$, then it is accelerated on crest through linac- 0 to $135 \mathrm{MeV}$. During the acceleration in linac-1, the bunch energy is chirped by shifting the rf phase to -25 degree. A $4^{\text {th }}$ harmonic X-band cavity is used to compensate the rf curvature effect in order to linearize the longitudinal phase space before the bunch compression. After passing through $\mathrm{BC} 1$ with the nominal momentum compaction $R_{56}=39 \mathrm{~mm}$, the rms bunch length is reduced to $0.19 \mathrm{~mm}$ with a compression ratio of about 4.4. Experimentally measured field data are adopted in most of the beam line elements such as the rf gun, the solenoid, and the dipole magnets used in the simulations.

\section{Space Charge Effects above $64 \mathrm{MeV}$}

After the first section of Linac-0, the electron energy is $64 \mathrm{MeV}$. We compared the simulation results with space charge on/off from this energy. Figure 1 shows the horizontal normalized emittance from the photocathode to DL1. It shows that the emittance can further decrease about 7 percent in horizontal direction when space charge effects are included. This can be explained by the fact that the solenoid field is optimized to compensate the space-charge induced emittance growth up to $135 \mathrm{MeV}$. While turning off the space charge forces at $64 \mathrm{MeV}$, the emittance compensation process is interrupted and the emittance is kept almost constant instead of decreasing to the fully compensated value. The vertical emittance evolution shows very similar behavior.

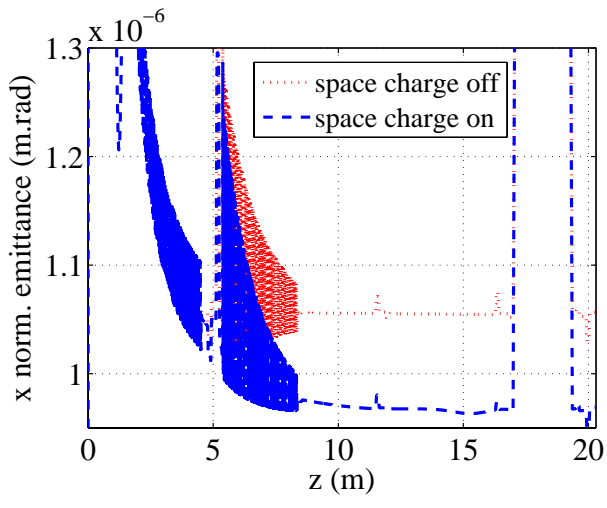

Figure 1: The horizontal normalized emittance with space charge effects on/off from energy of $64 \mathrm{MeV}$.

\section{Space Charge Effects above $135 \mathrm{MeV}$}

With this design setup, we study the space charge effects at the beam energy above $135 \mathrm{MeV}$ by switching on/off space charge calculation at the exit of linac- 0 . Simulations show that the difference on the transverse emittance between space charge on and off is less than 1 percent above this energy level, which is expected due to the $1 / \gamma^{2}$ space charge suppression factor. For a smooth temporal bunch profile the longitudinal space charge effect should also be negligible at such a high energy. Nevertheless, a temporally modulated bunch is subject to microbunching instability due to the longitudinal space charge force, which is discussed elsewhere $[12,13]$.

\section{SIMULATION STUDIES WITH BUNCH COMPRESSION}

The space charge effects can become important after the bunch compression when the electron bunch is fully compressed or when a current spike is formed through the nonlinear compression process as observed at the TTF FEL in [15]. We study an example of the space charge effects after $\mathrm{BC} 1$ that closely resembles the commissioning conditions as described in Ref. [1]. Using Impact-T, we simulate a bunch with 200-pC charge and 2.37-ps bunch length (rms). The longitudinal phase space and the slice energy spread at the injector end (at $135 \mathrm{MeV}$ ) are shown in Figure 2 . Note that the slice energy spread at the bunch center (with a peak current of $35 \mathrm{~A}$ ) is less than $1 \mathrm{keV}$. To speed up the simulation, we use Elegant [16] to model Linac-1 and $\mathrm{BC} 1$, including the linac wakefields and CSR but neglecting space charge effects in this part of the machine. We set the Linac-1 $\mathrm{rf}$ phase to 42 degree and use the nominal BC1 $R_{56}=39 \mathrm{~mm}$. In the absence of the X-band cavity that linearizes the longitudinal phase space before the compression, only the middle section of the bunch undergoes full compression. Nevertheless, a current spike of 5 $\mathrm{kA}$ is produced in the bunch head as shown in Figure 3. The full width of the spike is extremely narrow and may be estimated to be $4.8 R_{56} \sigma_{\delta} \approx 1 \mu \mathrm{m}$ [15]. A rough estima- 

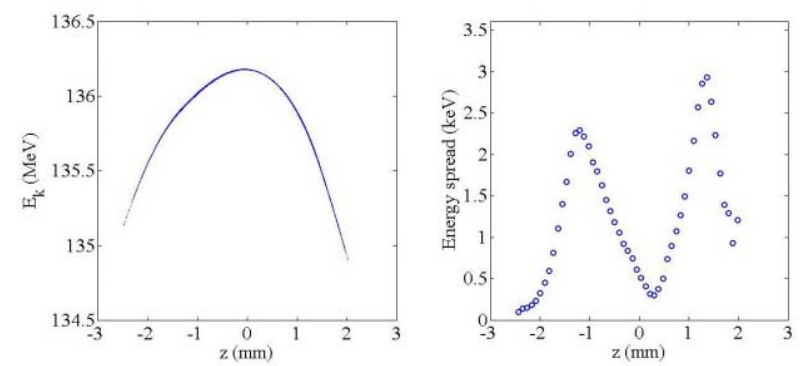

Figure 2: The longitudinal phase space (left) and the slice energy spread (right) at the exit of Linac-0. Bunch head at right.
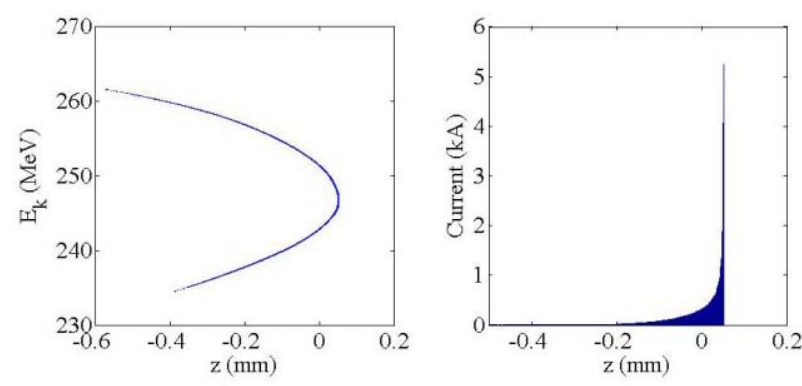

Figure 3: The longitudinal phase space (left) and the current profile (right) at the exit of $\mathrm{BC} 1$. Bunch head at right.

tion using these parameters from the standard longitudinal space charge formula predicts a strong space charge effect after this bunch transporting several meters, around $\mathrm{MeV}$ order.

Using Impact-T, we simulate a 3-m transport after BC1 to the location where emittance can be measured experimentally with the OTR diagnostic station. Figure 4 shows the longitudinal phase space at this location, where the energy of the current spike is strongly modified due to the presence of the longitudinal space charge force. Transverse space charge effects also increase the emittance in this spike, as shown in Fig. 5. Further studies are planned to quantified these effects in order to have a better understanding of the experimental results.

\section{SUMMARY}

The parallel implementation of 3-D space charge algorithm makes Impact- $T$ a powerful tool for high-brightness beam dynamics studies. In this paper, we focus on the relatively high-energy part of the LCLS injector and compressor system where the space charge effects have not been thoroughly studied before. Detailed comparison with the experimental results will be forthcoming.

\section{REFERENCES}

[1] P. Emma et al., these Proceedings, TUPMS049 and TUOCAB02, 2007.
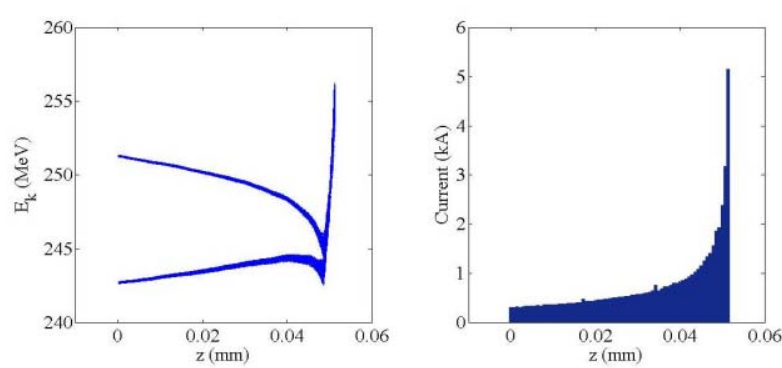

Figure 4: The head section of the longitudinal phase space (left) and the current profile (right) after 3-meter drift of $\mathrm{BC} 1$ exit. Bunch head at right.

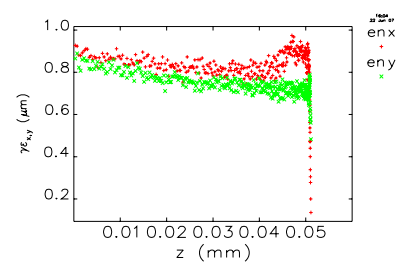

(a)

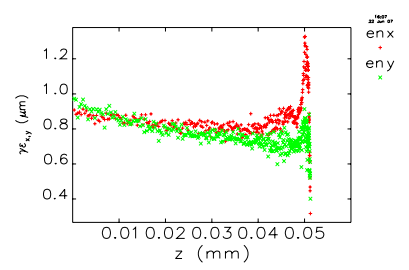

(b)
Figure 5: The slice emittance (a) at the exit of $\mathrm{BC} 1$, (b) at 3-m downstream of $\mathrm{BC} 1$. Bunch head at right.

[2] D.H. Dowell et al., Proceedings of EPAC'04, Luzern, July 2004.

[3] J. Qiang, S. Lidia, R. D. Ryne, C. Limborg-Deprey, Phys. Rev. Special Topics - Accel. Beams 9, 044204, (2006).

[4] J. Qiang et al., in Proceedings of PAC'05, Knoxville, Tennessee, 2005, p. 3316.

[5] K. L.F. Bane, SLAC-PUB-9663, 2003.

[6] P. Craievich, T. Weiland, I. Zagorodnov, ST/M-04/02, 2002.

[7] E. L. Saldin, E. A. Schneidmiller, and M. V. Yurkov, Nucl. Instrum. Methods Phys. Res., Sect. A398, 373 (1997).

[8] M. Borland, Phys. Rev. Sepecial Topics - Accel. Beams 4, 070701 (2001).

[9] G. Stupakov and P. Emma, SLAC-PUB-9242, 2002.

[10] G. A. Loew, R. H. Miller, R. A. Early and K. L. Bane, SLAC-PUB-2295 (1979).

[11] J. Qiang, IMPACT-T User Document Version 1.5, LBNL rep 62326, 2007.

[12] Z. Huang et al. Phys. Rev. ST Accel. Beams 7, 074401 (2004).

[13] I. V. Pogorelov et al. these Proceedings, TUPMN114 (2007).

[14] K. L.F. Bane, Phys. Rev. Sepecial Topics - Accel. Beams 5, 104401 (2002).

[15] M. Dohlus et al., Nucl. Instr. and Meth. A530, 217.

[16] M. Borland, ANL Advanced Photon Sourse Report No. LS287, 2000. 